\title{
Energy-Efficient Gaming on Mobile Devices using Dead Reckoning-based Power Management
}

R. Cameron Harvey, Ahmed Hamza, Cong Ly, Mohamed Hefeeda

Network Systems Laboratory

Simon Fraser University

November 16, 2010 


\section{Outline}

(1) Introduction

(2) Related Work

(3) Dead Reckoning

(4) Dead Reckoning Sleep (DRS) Algorithm

(5) Evaluation

6) Conclusions 


\section{Outline}

\section{(1) Introduction}

\section{Related Work}

Dead Reckoning

Dead Reckoning Sleep (DRS) Algorithm

\section{Evaluation}

\section{Conclusions}




\section{Mobile Gaming}

- Mobile gaming revenues are estimated to reach $\$ 1.5$ billion in the US by 2014 [eMarketer]

- 64 million people will play mobile games at least monthly, a number that will rise to 94.9 million by 2014

- Mobile gaming market is predicted to reach $\$ 18$ billion by 2014 (\%16.6 annual growth rate) [Pyramid Research]

- In 2010, factory unit shipments of game-capable mobile phones are forecasted to reach 1.27 billion [iSuppli Corp]

- In addition to commercially available games, many games have been ported to Android-based phones/devices (e.g. Kwaak3) 


\section{Motivation}

- Gaming uses a lot of power

- The screen is always on

- CPU used more intensively (calculations and rendering)

- Wireless network interface for communication

- Wireless network interface card can account for up to $70 \%$ of total power consumption in mobile devices

- Muliplayer games need to send state updates to maintain game state consistency among players

- Power Consumption vs. Consistency

- How can we reduce energy consumption of wireless interface without greatly affecting consistency? 


\section{Related Work}

\section{Outline}

\section{Introduction}

\section{(2) Related Work}

\section{Dead Reckoning}

Dead Reckoning Sleep (DRS) Algorithm

\section{Evaluation}




\section{Related Work}

- IEEE 802.11 Power Saving Mode (PSM)

- Only available in infrastructure mode

- Gaming traffic has real-time constraints [CC'6]

- Bounded-Slowdown

- Dynamically adapts sleep periods to past network activity

- Requires making changes to existing protocols and standards

- Minimize energy consumption by turning off the wireless interface [SBS'02] [ZMG'05]

- Scheduling algorithms to determine sleep periods

- Formulate a complex scheduling algorithm 


\section{Outline}

\section{Introduction}

\section{Related Work}

\section{(3) Dead Reckoning}

\section{Dead Reckoning Sleep (DRS) Algorithm}

\section{Evaluation}

\section{Conclusions}




\section{Dead Reckoning}

- Multiplayer games

- Avatar games (player controls a single character)

- first-person avatar: player's view is through the character's eyes

- third-person avatar: player sees the character from a distance

- Omnipresent games (player concurrently controls a group of characters)

- can interact with objects close to any of the characters

- include real-time strategy games and simulation games

- After agreeing on game settings (e.g. map and rules), players form a gaming session

- One client is chosen as the authoritative host (to maintain consistency) 


\section{Dead Reckoning}

- Dead Reckoning (DR)

- The process of estimating the future position of an object given its original position, intended course, velocity, and amount of time passed

- DR is used to hide network latency and reduce network traffic in multiplayer games

- Extrapolate behavior and state of gaming objects $\rightarrow$ can continue rendering frames even if game-state updates are late.

- A dead reckoning vector typically contains:

- Current position of the player (in terms of $x, y$, and $z$ coordinates)

- Velocity

- Clients agree on a predictive contract mechanism, and ensure the two models do not deviate beyond a threshold

- Dead reckoning error is the deviation between actual and extrapolated trajectories 


\section{Dead Reckoning}

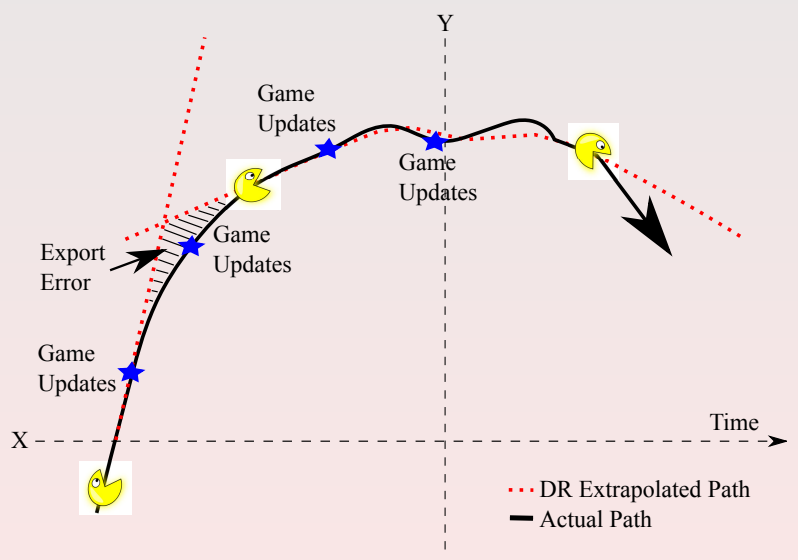

Figure: Dead reckoning 


\section{Potential Sleep Periods}

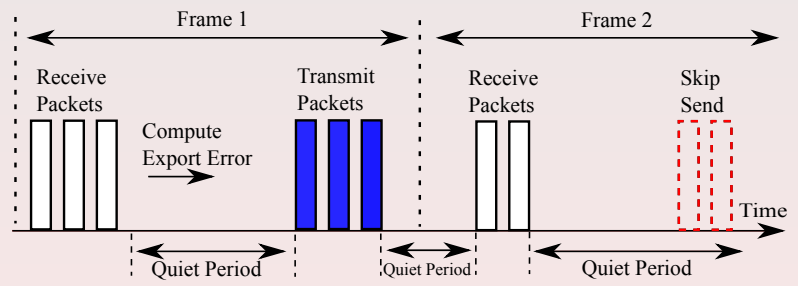

Figure: Game interactions with the wireless interface 


\section{Outline}

\section{Introduction}

\section{Related Work}

Dead Reckoning

(4) Dead Reckoning Sleep (DRS) Algorithm

\section{Evaluation}




\section{Dead Reckoning Sleep (DRS) Algorithm}

- Idea: exploit dead reckoning to predict periods of inactivity in the wireless device during game play

- Predict how long it will take before the next update will occur

- Based on how close the current DR error is to the threshold

- Divide threshold value for each DR variable into $n$ intervals

- Each interval has a corresponding storage bin for the statistical information used to predict when the wireless interface will be needed

- A bin maintains a weighted moving average for the time duration until threshold is exceeded

- If the receiver is sleeping, state updates are cached by authoritative server 


\section{Dead Reckoning Sleep (DRS)}

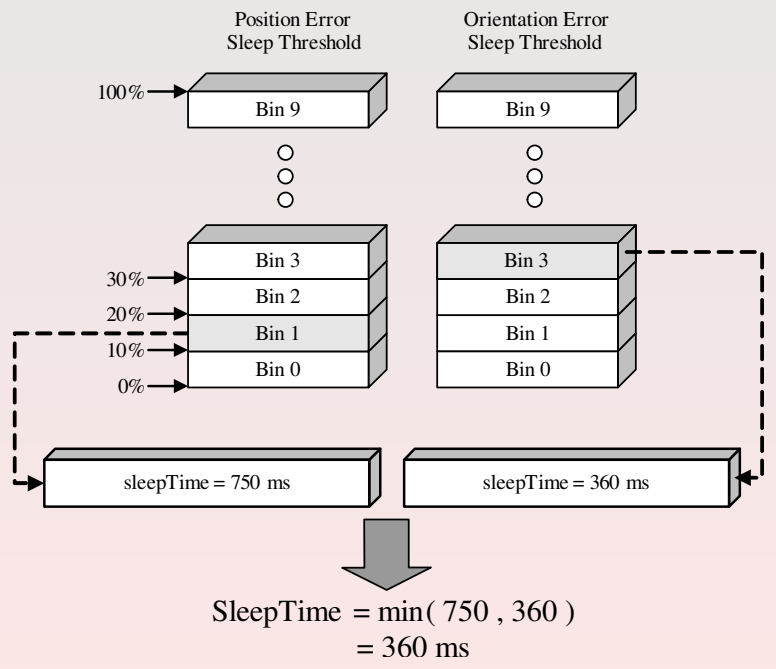

Figure: Threshold partitioning 


\section{Dead Reckoning Sleep (DRS) Algorithm}

\section{Estimated Sleep Time}

$$
\text { estST }_{i}=(1-\alpha) \cdot \text { estST }_{i}+\alpha \cdot\left(\text { currentlnterval }_{i}\right)
$$

\section{Variability Estimation}

$$
\operatorname{DevST}_{i}=(1-\beta) \cdot \operatorname{DevST}_{i}+\beta \cdot \mid e s t S T_{i}-\text { currentInterval }_{i} \mid
$$

\section{Sleep Time}

$$
\text { sleepTime }_{i}=\text { estST }_{i}-\gamma_{i} \cdot\left(\text { DevST }_{i}\right)
$$

- $\gamma$ : conservative offset factor to mitigate the variability and to ensure we do not sleep too long 


\section{Input: $N$ : Number of DR variables}

Input: error[], threshold[]: DR errors and thresholds

Input: PSP: Power saving profile

Input: Wireless state

Input: $Q$ : Queue for DR error bins

for $i \leftarrow 0$ to $N-1$ do

if error $[i]<$ threshold $[i]$ then

Add bin corresponding to error $[i]$ to $Q$;

sleepTime $[i] \leftarrow 0$;

else

Update weighted averages of queued bins;

Empty $Q$;

if wireless is sleeping then

I Wake wireless;

else

I Send update;

end

end

end

Put wireless to sleep for $P S P \cdot \min _{0 \leq i \leq N-1}($ sleepTime $[i])$; 


\section{Outline}

\section{Introduction}

\section{Related Work}

\section{Dead Reckoning}

\section{Dead Reckoning Sleep (DRS) Algorithm}

\section{(5) Evaluation}




\section{Evaluation of DRS Algorithm}

- Modify the Game Latency Simulator (GLS) from University of Oslo

- Wireless controller module which implements DRS

- Power consumption model based on the characteristics of Cisco AIR-PCM350

- Simulate a two hour game session between two players

- Chosen values for $\alpha$ and $\beta$ are $0.125,0.25$, respectively

- Defaults:

frame duration $=40 \mathrm{~ms}, \mathrm{PSP}=1.0$, granularity $=10$, threshold factor $=0.8$

- Evaluation Metrics

- Energy savings, average estimation error, and average position deviation. 


\section{BZFlag}

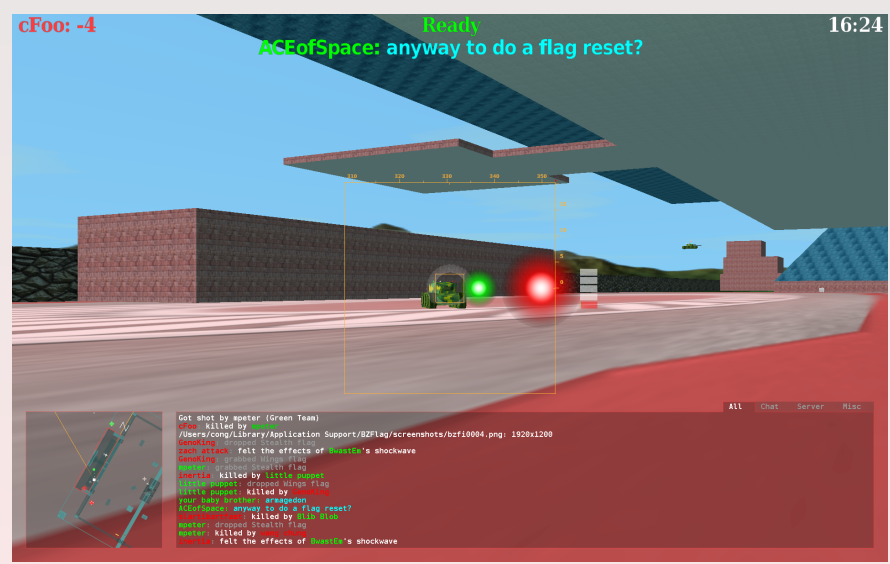

Figure: Screen capture from BZFlag 


\section{Game Latency Simulator}

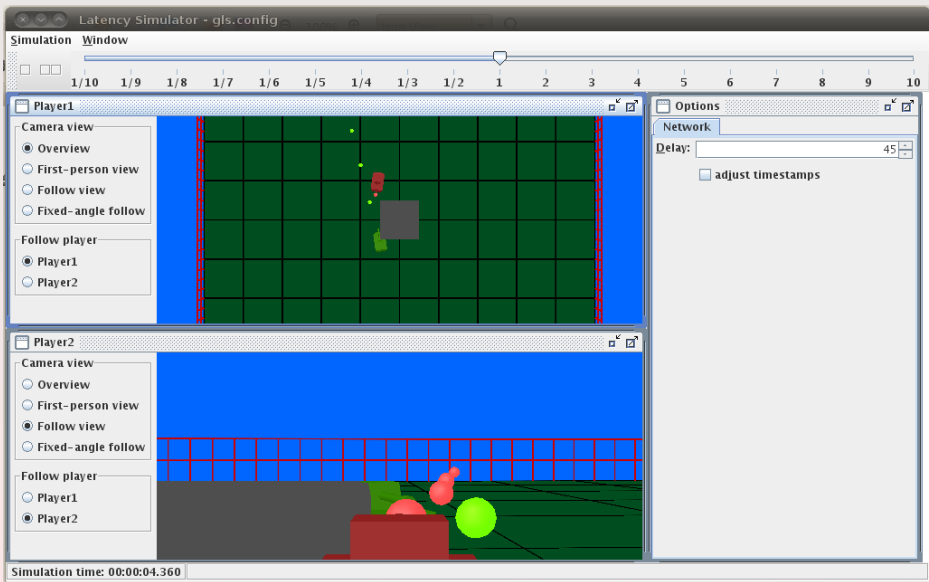

Figure: Screen capture from GLS 


\section{Gamma Effect}
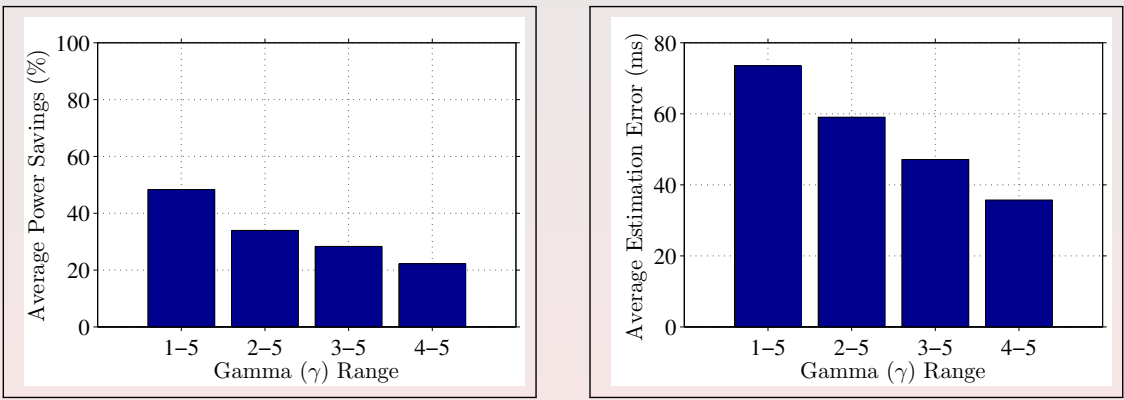

- Tradeoff:

- Wider $\gamma$ range $\rightarrow$ more power savings

- Narrower $\gamma$ range $\rightarrow$ fewer estimation errors 


\section{Gamma Effect}

- About $60 \%$ of the estimation errors are $300 \mathrm{~ms}$ or less

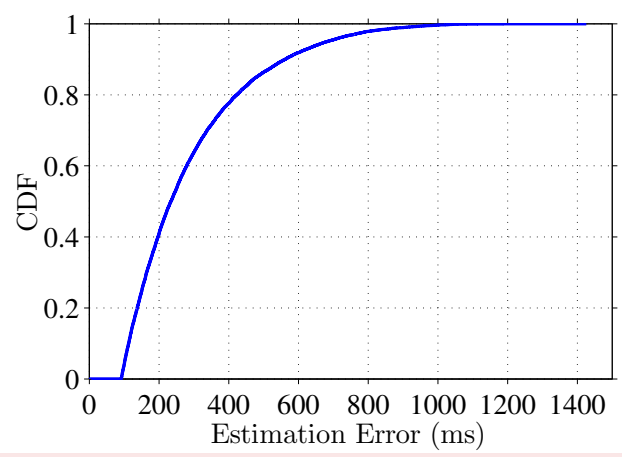

Figure: Cummulative Distribution Function of Estimation Errors $(\gamma: 3 \rightarrow 5)$ SFU 


\section{Power Savings}

- Energy savings are more pronounced at higher frame rates

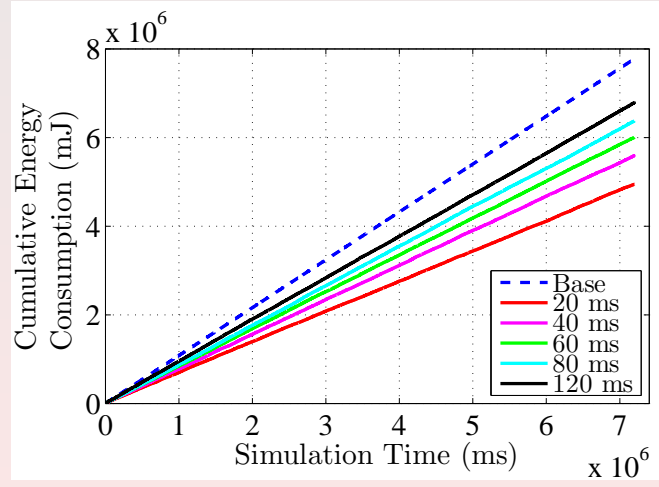

Figure: Cumulative energy consumption at various frame durations 


\section{Average Sleep Time Estimation Errors}
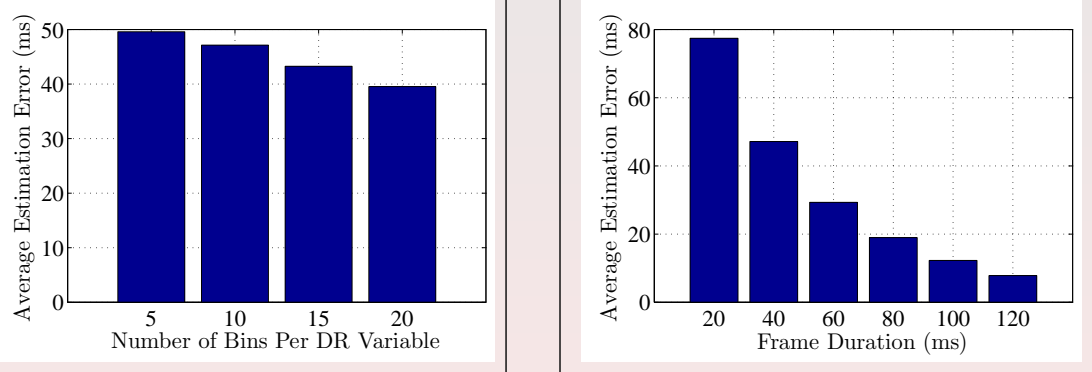

- Average sleep time estimation error increases almost exponentially as the framerate is increases

- Higher framerates $\rightarrow$ sleep durations span more frames, with the first frame being closer to the beginning of the sleep cycle 


\section{Average Position Deviation}

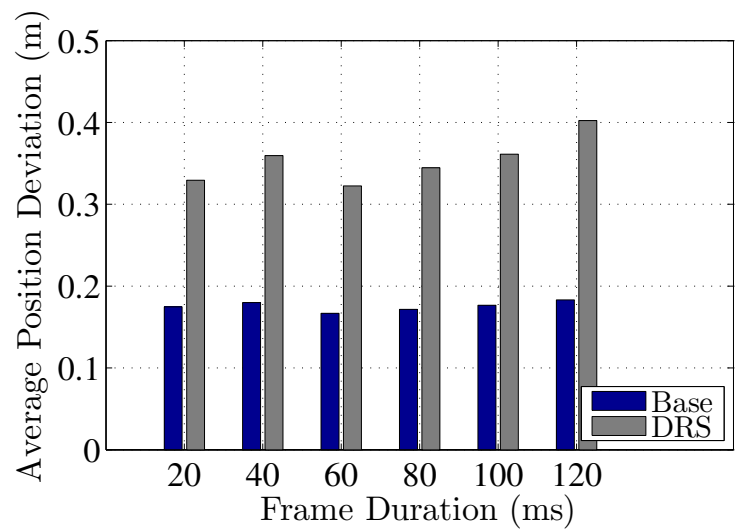

Figure: Average position deviation vs. frame duration 


\section{Average Position Deviation}

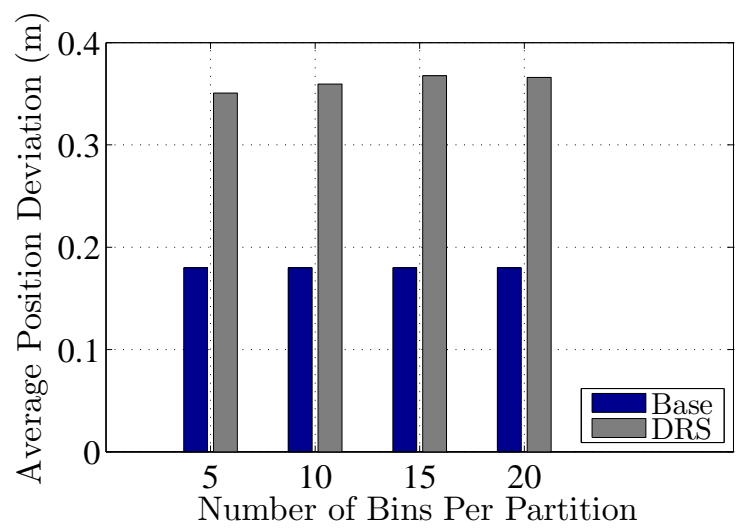

Figure: Average position deviation vs. granularity of partitions 


\title{
Outline
}

\section{Introduction}

Related Work

Dead Reckoning

Dead Reckoning Sleep (DRS) Algorithm

\author{
Evaluation
}

(6) Conclusions 


\section{Conclusions}

- Mobile gaming is gaining popularity with a rapidly growing market

- Wireless network interface is one of the main sources of power drain in mobile devices

- Proposed a new power saving algorithm utilizing dead reckoning to predict wireless interface sleep cycles

- Simulation results show that power savings up to $36 \%$ can be achieved in most gaming sessions using the DRS algorithm

- Power savings come at some cost in terms of game state consistency 


\section{Future Work}

- Study implications of cheating during game play on power management algorithms

- Develop a testbed and implement DRS into the BZFlag code

- Extend our implementation to mobile devices such as the Google Nexus One phone 


\section{References}

圆 [SBS'02]

E. Shih, P. Bahl, and M. J. Sinclair, Wake on wireless: an event driven energy saving strategy for battery operated devices, MobiCom'02, 2002.

[ZMG'05]

T. Zhang, S. Madhani, P. Gurung, and E. van den Berg, Reducing energy consumption on mobile devices with WiFi interfaces, GLOBECOM'05, 2005.

圆 [CC'06]

M. Claypool and K. Claypool, Latency and player actions in online games, Communications of the ACM, 2006. 


\section{Thank You}

\section{Questions?}

